\title{
Metastable Amphiphilic Hydrogels Based on Crosslinked Carboxymethylpullulan
}

\author{
By Mélanie Legros, ${ }^{1}$ Pascal CARDinael, ${ }^{2}$ Virginie Dulong,${ }^{1}$ Luc PICTON, ${ }^{1}$ and Didier Le CERF ${ }^{1, *}$
}

Crosslinked carboxymethylpullulan have been synthesized by using the dibromohexan as a crosslinking agent. Two phases are obtained after synthesis: a gel and a sol fraction. The sol/gel ratio is dependent on the variation of the polymer concentration $\left(\mathrm{C}_{\mathrm{p}}\right)$, the incorporation ratio of the dibromohexan $\left(\mathrm{R}_{\mathrm{c}}\right)$ and the average molar mass $\left(M_{\mathrm{n}}\right)$. The study of the swelling degree in water of the gel fraction has shown that this phase is not stable and that it shrinks after a certain time. This phenomenon is accelerated by an increase in temperature. At a macroscopic level, the gelation point is not reached. Nevertheless, the quantity of dibromohexan incorporated seems to be sufficient to obtain a gel.

KEY WORDS: Crosslinked Carboxymethylpullulan / Dibromohexan / Metastable / Nanogels /

Amphiphilic polysaccharides have attracted much attention in recent years to replace their synthetic homologues. Indeed, most of them are biodegradable and biocompatible, so they can find a huge number of applications, especially in pharmaceutical and biomedical fields. As a general rule, they are used in formulation as texture agent, but they can also be utilized in drug delivery domains. In the literature, some examples of surfactants based on amphiphilic polysaccharides are reported. Generally, they are needed either to stabilize particles or to modify the surface properties of colloidal particles charged with drugs or proteins. ${ }^{1-6}$

Amphiphilic polysaccharides can also be used directly as carrier molecules. Some studies have been interested in forming macroscopic hydrogels by self-associations of hydrophobic moieties in water. ${ }^{7-9}$ But from some years, several works have been conducted to develop nanogels, which are easily assimilated by living organisms. Akiyoshi et al. have elaborated nanometric hydrogels formed of self-aggregated cholesterol-bearing pullulan able to complex several proteins and to release them. ${ }^{10-13}$ Whereas $\mathrm{Na}$ et al. have used selfassembled nanoparticles to deliver specifically drugs into tumor cells. ${ }^{14}$ We can also find examples of chemical nanogels. Vinogradov et al. have developed nanogels based on crosslinked poly(ethyleneglycol) and polyethylenimine able to encapsulate and deliver oligonucleotides in their targets in the cell. ${ }^{15-17}$ But these samples are obtained from hydrophilic, synthetic polymers. In fact, only few studies have been interested in chemical hydrogels based on amphiphilic polysaccharides, at a macroscropic or nanometric scale, because it is difficult to crosslink these macromolecules after their hydrophobization. To solve this problem, Dulong et al. have used an amphiphilic crosslinking agent: the adipic acid dihydrazide, which brings at the same time the amphiphilic character and the crosslinks. ${ }^{18}$ Other molecules are able to act like adipic acid dihydrazide, especially a large part of the dihalogenoalcan. Coviello et al. have used the dibromohexan to crosslink scleroglucan chains, but, in spite of the amphiphilic feature of their crosslinking agent, they have not studied this particular aspect. ${ }^{19,20}$

Here, we have used the dibromohexan to crosslink a derivative of the pullulan, the carboxymethylpullulan (CMP), with the intention of creating systems able to entrap hydrophobic or amphiphilic compounds.

The carboxymethylpullulan is obtained from pullulan, a neutral, flexible exopolysaccharide according to a reaction described elsewhere. ${ }^{21}$ Several studies have been conducted in our team to hydrophobize the CMP, especially with bromoalcans via ester linkage. ${ }^{22}$ We have been inspired by this hydrophobization to realize our crosslinking reaction by replacing the bromoalcan by a dibromoalcan.

In this paper, we report first on the synthesis of the crosslinked carboxymethylpullulan as a function of some parameters such as polymer and crosslinking agent concentrations, molar mass of CMP. Then, the quantity of Alcan in final product has been determined with a particular attention about mono and bi incorporation of the dibromoalcan onto the polysaccharide. The gel has been characterized by its swelling properties in water in function of time and temperature. Finally we discuss on the origin of gel-sol transition.

\section{MATERIALS AND METHODS}

\section{Materials}

Pullulan $\left(\bar{M}_{\mathrm{n}}=120000 \mathrm{~g} \cdot \mathrm{mol}^{-1} ; \bar{M}_{\mathrm{w}}=280000 \mathrm{~g} \cdot \mathrm{mol}^{-1}\right.$ determined by Size Exclusion Chromatography coupled with Multi Angle Laser Light Scattering (SEC/MALLS)) was purchased from Hayashibara Biochemical Laboratory (Okayama, Japan). Isopropanol, dimethylsulfoxide (DMSO), hexanol, acetonitrile, sodium chloroacetate, sodium hydroxide, hydrochloric acid, tetrabutylammonium hydroxide, dibromohexan and sodium azide were purchased from Acros Organics. Sodium chloride, lithium nitrate and acetone were purchased 


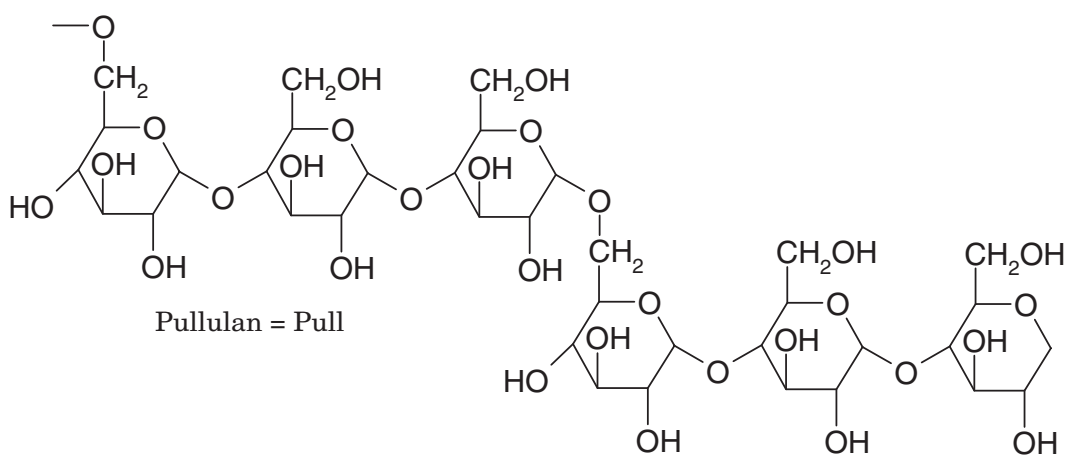

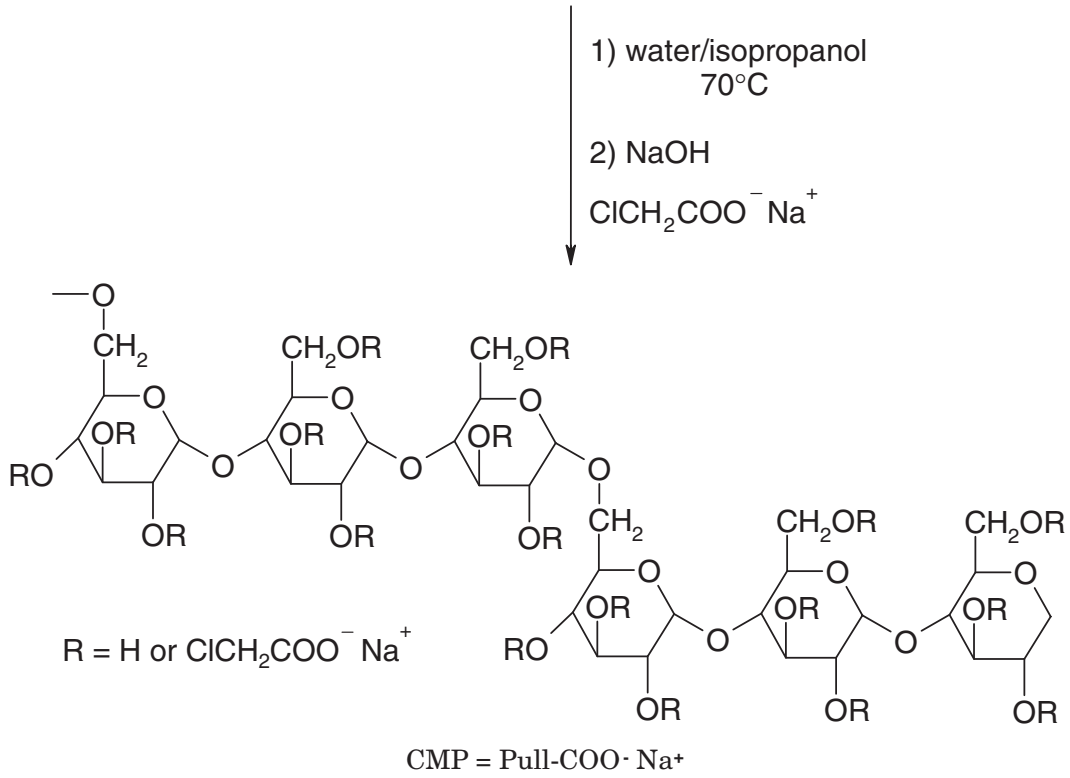

Figure 1. Carboxymethylation of pullulan.

Table I. Characteristics of pullulan and CMP derivatives $\mathrm{Ip}=\bar{M}_{\mathrm{w}} / \bar{M}_{\mathrm{n}}$

\begin{tabular}{|c|c|c|c|c|c|c|}
\hline Samples & $\mathrm{DS}^{\mathrm{a}}$ & $\bar{M}_{\mathrm{n}}^{\mathrm{b}}\left(\mathrm{g} \cdot \mathrm{mol}^{-1}\right)$ & $\bar{M}_{\mathrm{w}}^{\mathrm{b}}\left(\mathrm{g} \cdot \mathrm{mol}^{-1}\right)$ & $I p^{b}$ & $\mathrm{M}_{\text {unit }}\left(\mathrm{g} \cdot \mathrm{mol}^{-1}\right)$ & $\mathrm{DP}_{\mathrm{n}}$ \\
\hline Pullulan & 1 & 120000 & 280000 & 2.3 & 162 & 741 \\
\hline HMCMP1 & 0.78 & 90000 & 200000 & 2.2 & 224.4 & 401 \\
\hline HMCMP2 & 1.13 & 89000 & 230000 & 2.6 & 252.4 & 353 \\
\hline HMCMP3 & 0.96 & 150000 & 310000 & 2.1 & 238.8 & 628 \\
\hline MMCMP2 & 1.13 & 56000 & 106000 & 1.9 & 252.4 & 222 \\
\hline MMCMP3 & 0.96 & 52000 & 79000 & 1.5 & 238.8 & 218 \\
\hline LMCMP2 & 1.13 & 15000 & 27000 & 1.8 & 252.4 & 59 \\
\hline LMCMP3 & 0.96 & 23000 & 34000 & 1.5 & 238.8 & 96 \\
\hline
\end{tabular}

adetermined by conductimetry, ${ }^{b}$ determined by SEC/MALLS.

from VWR Prolabo. Hexanediol was obtained from Aldrich. All reagents and solvents were used without further purification. Water was purified with a Milli-Q water reagent system (Millipore).

\section{Synthesis of Carboxymethylpullulan}

Sodium salt of carboxymethylpullulan $\left(\mathrm{CMP}, \mathrm{Na}^{+}\right)$was synthesized in water/isopropanol medium by reacting the hydroxyl groups of pullulan with sodium chloroacetate in the presence of sodium hydroxide (Figure 1) according to a procedure described previously. ${ }^{21}$
The degree of substitution (DS) of CMP, which corresponds to the number of carboxymethyl group per anhydroglucose unit, was determined by conductimetric titration according to the Eyler's ${ }^{23}$ method and the average molar masses were determined by SEC/MALLS measurements (Table I).

\section{Degradation of Carboxymethylpullulan}

$2.5 \mathrm{~g}$ of carboxymethylpullulan was dissolved in $500 \mathrm{~mL}$ of milli-Q water. After complete dissolution, the $\mathrm{pH}$ of the solution was set to 2 by adding $\mathrm{HCl} 4 \mathrm{M}$. Then, this solution was heated at $60{ }^{\circ} \mathrm{C}$ under magnetic stirring during $5 \mathrm{~d}$ for a 
medium degradation or during $14 \mathrm{~d}$ for a very high degradation. The average molar masses of the degraded carboxymethylpullulan were checked by SEC/MALLS (Table I). We also verified by conductimetric measurements that the DS was not changed during the degradation.

\section{Synthesis of Crosslinked Carboxymethylpullulan}

Crosslinked carboxymethylpullulan was obtained by a synthetic pathway adapted from that used by Duval et al. to hydrophobize the carboxymethylpullulan. ${ }^{22}$

An aqueous solution of CMP, $\mathrm{Na}^{+}\left(25 \mathrm{~g} \cdot \mathrm{L}^{-1}\right)$ was eluted through a cationic resin (Amberlite IRN-77, $\mathrm{H}^{+}$form) to obtain the acidic form of CMP $\left(\mathrm{CMP}, \mathrm{H}^{+}\right)$. This solution was neutralized by tetrabutylammonium hydroxide $\left(\mathrm{Bu}_{4} \mathrm{~N}^{+}, \mathrm{OH}^{-}\right)$ up to $\mathrm{pH} 7$ and freeze-dried. Then, $\mathrm{CMP}, \mathrm{Bu}_{4} \mathrm{~N}^{+}$was dissolved at various concentrations in DMSO at $40{ }^{\circ} \mathrm{C}$ during $12 \mathrm{~h}$. A known amount of dibromohexan was introduced under vigorous stirring to obtain a given theoretical incorporation ratio, $\mathrm{R}_{\mathrm{c}}$ defined as the molar ratio of dibromohexan on the carboxylic groups per AGU. The reaction was continued at $40{ }^{\circ} \mathrm{C}$ under magnetic stirring for $2 \mathrm{~h}$. The products obtained were first dialyzed against water until complete removal of DMSO (checked by conductimetry), then against $\mathrm{NaCl} 1 \mathrm{M}$ to exchange the tetrabutylammonium ions against sodium ones and finally against water to remove the excess of sodium salt (checked by conductimetric measurements). The samples were filtered to separate the gel fraction from the sol fraction. The sol fraction was freeze-dried, whereas the gel was precipitated in acetone and dried in a vacuum oven at $40{ }^{\circ} \mathrm{C}$.

\section{Characterization of the Crosslinked Carboxymethyl- pullulan}

Yields of Gel and Sol Fractions. After drying or freeze-drying, the gel and the sol fractions were weighted and the yields of gel and sol were calculated according to the equations (1) or (2):

$$
\begin{gathered}
\%_{\text {gel } r}=\left(\frac{W_{\text {gel }}}{W_{\text {gel }}+W_{\text {sol }}}\right) \times 100 \\
\%_{\text {sol } r}=\left(\frac{W_{\text {sol }}}{W_{\text {gel }}+W_{\text {sol }}}\right) \times 100
\end{gathered}
$$

where $W_{\text {gel }}$ and $W_{\text {sol }}$ are respectively the weight of gel fraction and the weight of sol fraction.

The uncertainty was measured to $5 \%$.

Incorporation Yield of Crosslinking Agent. The ratio of dibromohexan really incorporated in the samples was determined by quantifying the hexanediol released after total alkaline hydrolysis: $0.1 \mathrm{~g}$ of polymer was left to react in 30 $\mathrm{mL}$ of $5 \mathrm{M} \mathrm{NaOH}$ for $24 \mathrm{~h}$ at $25^{\circ} \mathrm{C}$. All the water was removed by evaporation and $30 \mathrm{~mL}$ of isopropanol containing a known amount of hexanol as an internal reference were added. The mixture was shacked during $4 \mathrm{~h}$ and filtered. The recovered solution containing the released hexanediol was finally analysed by gas phase chromatography (HP 6890 Series GC System; capillary column Optima FFAP $30 \mathrm{~m} \times 250 \mu \mathrm{m} \times$ $0.25 \mu \mathrm{m}$; injection temperature $230{ }^{\circ} \mathrm{C}$; column temperature $80^{\circ} \mathrm{C}$ then increase up to $230^{\circ} \mathrm{C}$ at $10^{\circ} \mathrm{C} \cdot \mathrm{min}^{-1}$ and decrease to $80^{\circ} \mathrm{C}$ at $10^{\circ} \mathrm{C} \cdot \mathrm{min}^{-1}$; detector temperature $250{ }^{\circ} \mathrm{C}$; helium flow 15.2 psi). Retention times were $2.1 \mathrm{~min}$ for isopropanol, $4.9 \mathrm{~min}$ for hexanol and $14.2 \mathrm{~min}$ for hexanediol. Concentrations of hexanediol were obtained from the peak areas. The real percentage of incorporation of the crosslinking agent in the CMP was calculated according to the equation (3):

$$
I \mathrm{c}=\left(\frac{n_{\text {hexanediol }}}{n_{\mathrm{AGU}} \times D S}\right) \times 100
$$

where $n_{\text {hexanediol }}$ and $n_{\mathrm{AGU}}$ are respectively the mole number of hexanediol and the mole number of anhydroglucose (AGU) unit in the CMP, with an uncertainty of $10 \%$.

Elementary Analysis. The percentage of brome in our samples was determined by elementary analysis with an ARL 3580 Inductively Coupled Plasma atomic Emission Spectrophotometer (ICP-ES) at the "Service central d'analyse" of the Centre Nationale de la Recherche Scientifique (CNRS) in Vernaison (France).

Swelling of Gels. The swelling degree $(S W)$ of the gels was measured in water as a function of time and temperature. It was calculated according to equation (4) where $W_{\mathrm{t}}$ is the weight of the swollen gel at time and $\mathrm{w}_{0}$ the weight of the dried gel:

$$
S W=\frac{W_{\mathrm{t}}-W_{0}}{W_{0}}
$$

The uncertainty was evaluated to $5 \%$.

For this purpose, a known amount of dried gel was added to a large volume of water. At different time, the gel was filtered, weighted and put again in water. This experiment was conducted at different temperature i.e. $4{ }^{\circ} \mathrm{C}, 20^{\circ} \mathrm{C}, 40^{\circ} \mathrm{C}$ and $60{ }^{\circ} \mathrm{C}$.

\section{RESULTS AND DISCUSSION}

\section{Synthesis of Crosslinked Carboxymethylpullulan}

Crosslinked carboxymethylpullulan is synthesized in two steps. First, pullulan is modified to obtain carboxymethylpullulan (Figure 1). This modification introduces anionic charges on the polysaccharide backbone. This function is particularly more reactive than hydroxyl groups. ${ }^{22}$

Then, the crosslinking reaction is achieved by coupling the dibromohexan with the $\mathrm{CMP}, \mathrm{Bu}_{4} \mathrm{~N}^{+}$(Figure 2).

In fact, we observe the appearance of a gel less than five minutes after the introduction of the crosslinking agent. This rapidity presents a very important disadvantage: dibromohexan reacts immediately in the zone where it arrives, creating visible heterogeneities in the gel. In spite of a vigorous stirring, we have only achieved to reduce these heterogeneities and not to avoid them. In the literature, we can find some examples of rapidly formed gels: PEO-cl-PEI nanogels are formed in only 3-5 min whereas gels of partially acetylated chitosan and glutaraldehyde are obtained after approximately 5 min. ${ }^{24,25}$ But, they still homogeneous. We have to note that the products used in these examples are hydrophilic. So, we can think that our heterogeneities are caused by the hydrophobic character of our system. 


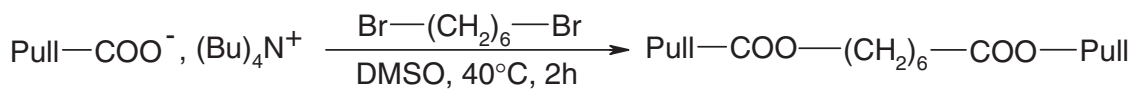

Figure 2. Synthesis of crosslinked carboxymethylpullulan.

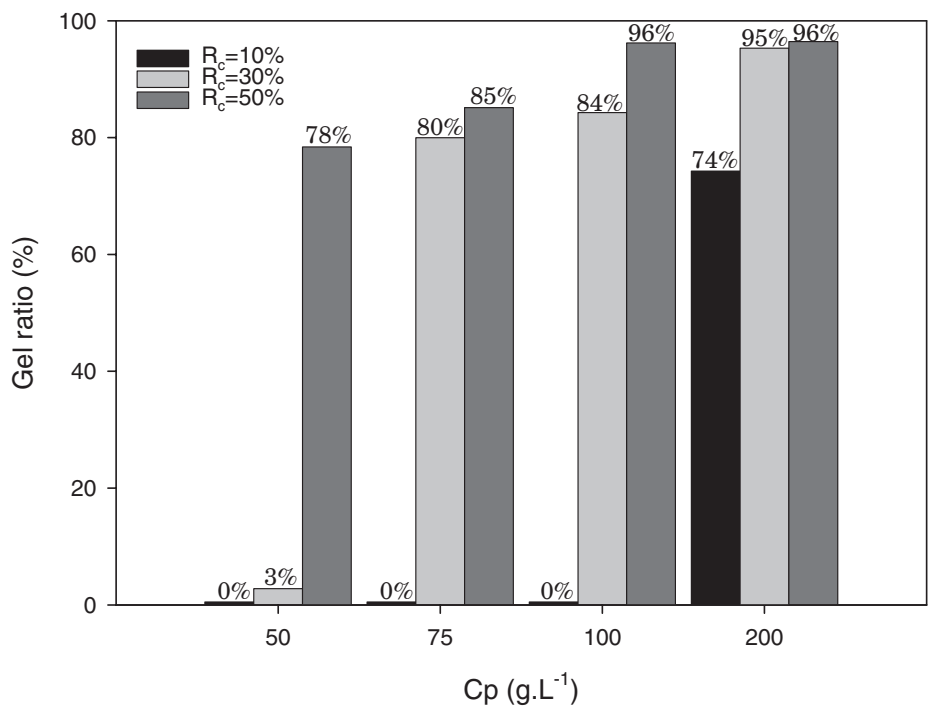

Figure 3. Influence of the polymer concentration on gel ratio of the crosslinked carboxymethylpullulan for $\bar{M}_{\mathrm{n}}=90000 \mathrm{~g} \cdot \mathrm{mol}{ }^{-1}$.

On the other hand, the purification of the samples is constituted by a series of dialyses against various solvents to eliminate the unreacted products and to regenerate the sodium carboxylate functions.

After this step, gel and sol fractions are separated by filtration on a linen cloth, which had pores of diameter about $33 \mu \mathrm{m}$. The gels obtained, whatever the synthesis conditions, are fragile, brittle and difficult to handle.

Finally, the sol fraction is freeze-dried whereas the gel fraction is precipitated in acetone and dried in a vacuum oven at $40^{\circ} \mathrm{C}$.

During this study, we have varied several parameters as the polymer concentration $\left(\mathrm{C}_{\mathrm{p}}\right)$, the concentration of dibromohexan and so the incorporation ratio of crosslinking agent $\left(\mathrm{R}_{\mathrm{c}}\right)$, the average molar mass of the CMP $\left(\bar{M}_{\mathrm{n}}\right)$ and the DS of the CMP.

In the following, samples will be coded by: $\mathrm{XMR}_{\mathrm{c}}-\mathrm{C}_{\mathrm{p}}$ with XM: the molar mass of the CMP (LM: Low Mass, MM: Medium Mass, HM: High Mass), $\mathrm{R}_{\mathrm{c}}$ : the theoretical incorporation ratio of dibromohexan $(\%)$ and $\mathrm{C}_{\mathrm{p}}$ : the polymer concentration $\left(\mathrm{g} \cdot \mathrm{L}^{-1}\right)$.

\section{Qualitative Aspect of Gel and Sol Fractions}

The ratios of gel and sol fractions vary as a function of the synthesis parameters i.e. the polymer concentration, the dibromohexan concentration and the average molar mass of the CMP. The following results will only present the ratios of the gel fractions, as the sol ratio is complementary.

For samples obtained with HMCMP1, the gel ratio increased with the polymer concentration whatever the incorporation ratio of dibromohexan was (Figure 3 ). The same results were observed with a CMP of medium molar mass (MMCMP2) (Figure 4). This can be explained by the increase of polymer chains entanglements, which facilitate the crosslinking reactions, with the increase of polymer concentration. Furthermore, the alkyl chain of the dibromohexan is relatively short, so if the chains are closer, the crosslinks' formation will be easier.

We have observed for most of the incorporation ratio that a threshold polymer concentration exists below which no gelation was observed (Figure 3). This critical concentration was all the more important as the quantity of dibromohexan was low. This behaviour was not obvious with the MMCMP, but more concentrated polymer solutions were needed to obtain gel fractions. In the case of $\mathrm{R}_{\mathrm{c}}=10 \%$, no gel was obtained: the polymer concentration was not sufficient to reach the gelation point.

We have reported in Figure 5 the evolution of the gel ratio versus the average molar mass and we observed also a critical value of $\bar{M}_{\mathrm{n}}$ below which no gel fraction was obtained when $\mathrm{C}_{\mathrm{p}}$ and $R_{c}$ are fixed. It is more difficult to have entanglements with shorter chains than with longer ones.

Another parameter could intervene in the sol/gel ratio: the substitution degree (DS) of the CMP. We have compared two samples of HM30-200 obtained from HMCMP with DS of 0.78 and 0.96 and we have found the same gel ratio of about $95 \%$. So we have deduced that, in this range, the number of carboxylate functions in the CMP does not influence the gelation.

From this study of the sol/gel ratio, we have concluded that the most important parameter in the gelation of our samples was the entanglement's level of the CMP's chains. 


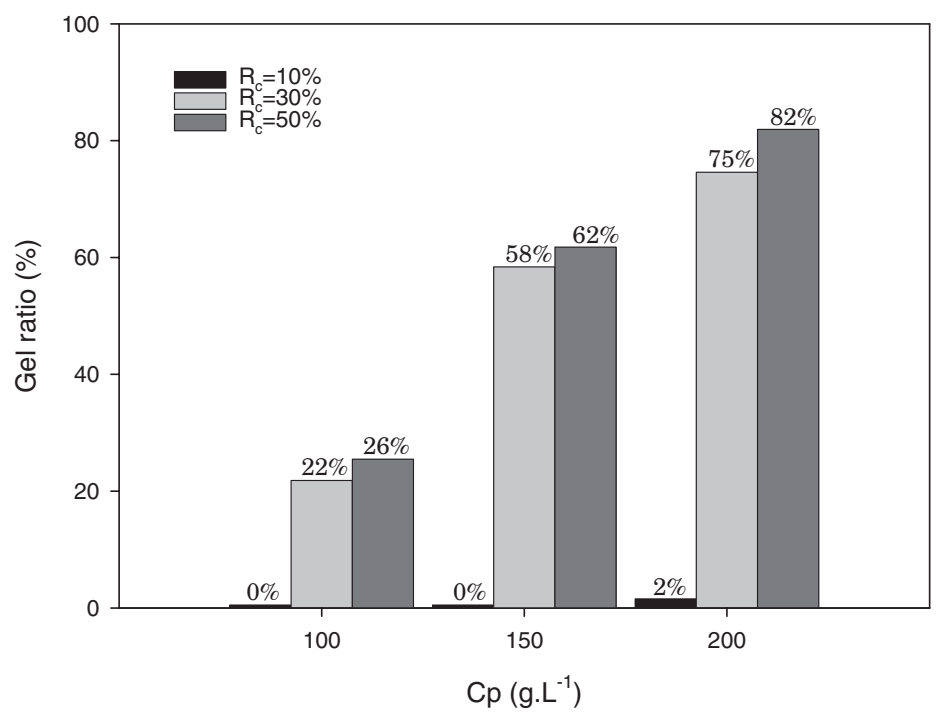

Figure 4. Influence of the polymer concentration on gel ratio of the crosslinked carboxymethylpullulan for $\bar{M}_{\mathrm{n}}=56000 \mathrm{~g} \cdot \mathrm{mol}{ }^{-1}$.

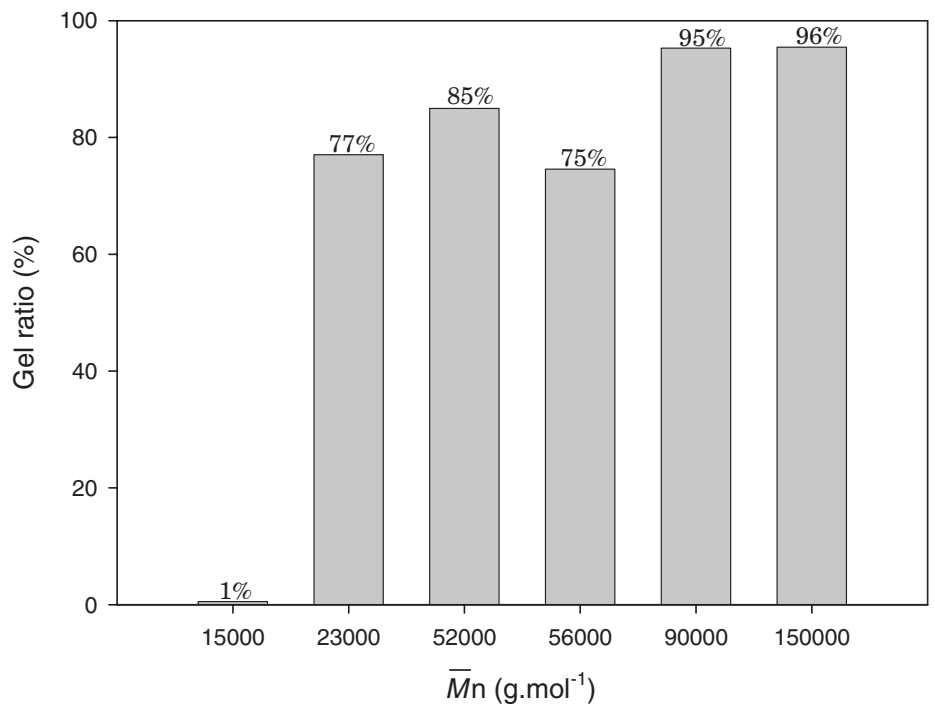

Figure 5. Influence of the average molar mass $\left(\bar{M}_{\mathrm{n}}\right)$ on gel ratio of the crosslinked carboxymethylpullulan for $C_{p}=200 \mathrm{~g} \cdot \mathrm{L}^{-1}$ and $\mathrm{R}_{\mathrm{c}}=30 \%$.

\section{Chemical Characterization of the Gel Fraction}

In order to know if the crosslinking reaction was effective, we had to determine the real incorporation ratio of dibromohexan and the way it reacted on the CMP.

For this purpose, we chose five representative samples for the following studies: HM30-200, HM30-100, HM30-50, MM30-200 and LM30-200, and especially their gel fractions.

First of all, we determined the quantity of dibromohexan incorporated in our systems to know if it was sufficient to crosslink the polysaccharide chains. This determination was realized after the complete hydrolysis of the ester linkage by measuring the hexanediol released with a gas phase chromatograph. As seen in Table II, the yield of the reaction for most of the samples was high $(\sim 70 \%)$, except for the LM30-200, which had incorporated only half of the crosslinking agent. So the calculated incorporation ratio of dibromohexan was about
Table II. Incorporation ratio of dibromohexan in the five representative samples $\mathrm{R}_{\mathrm{c}}$ : theoretical incorporation ratio of dibromohexan (\%) $\mathrm{I}_{\mathrm{C}}$ : experimental incorporation ratio of dibromohexan (\%)

\begin{tabular}{cccc}
\hline Sample & $\mathrm{R}_{\mathrm{c}}(\%)$ & $\mathrm{I}_{\mathrm{c}}(\%)$ & Yield of reaction (\%) \\
\hline HM30-200 & 30 & 23 & 74 \\
HM30-100 & 30 & 24 & 78 \\
HM30-50 & 30 & 22 & 69 \\
MM30-200 & 30 & 23 & 73 \\
LM30-200 & 30 & 17 & 54 \\
\hline
\end{tabular}

$20 \%$ for the majority of the samples and about $17 \%$ for the LM30-200. This type of analysis permitted us to reach the quantity of dibromohexan incorporated in the CMP, but not to know how it was incorporated. Indeed, the dibromohexan could react by its two brome extremities to give crosslinks, but 


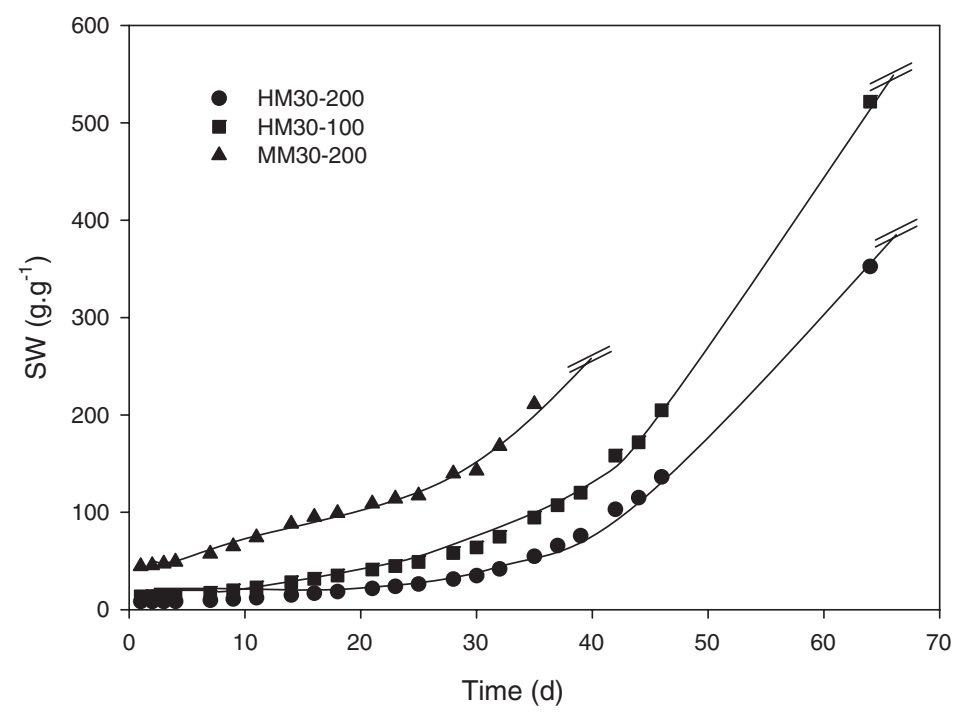

Figure 6. Kinetics of swelling of the gel fractions in water at $T=20^{\circ} \mathrm{C}$.

it can also react by only one brome function to give grafted alkyl chains on the CMP.

To know if we had grafted alkyl chains with a brome at the end, we used elementary analysis on two samples: HM30-200 and HM30-50. For these samples, we found very weak trace of brome $(<0.2 \%)$. But it was possible that the brome was hydrolysed and that we obtained alkyl chains with a hydroxyl function at the end.

In a first time, we thought to use NMR analyses to determine the presence of grafted pendant chains, but in ${ }^{1} \mathrm{H}$ NMR as well as in ${ }^{13} \mathrm{C}$ NMR, we had difficulties to distinguish the different peaks because of the numerous hydroxyl functions on the polysaccharide.

So we used another technique to determine if we had alkyl pendant chains. We titrated the unreacted carboxylate functions of a HM30-50 by conductimetry according to the Eyler's method. ${ }^{23}$ This sample had the greatest probability to have alkyl pendant chains; indeed after synthesis, it had only a sol fraction. These measurements permitted us to know the quantity of carboxylate functions which had reacted, and in comparison with the results obtained in GPC, we found that one dibromohexan had reacted with two carboxylate functions. So, if in the less favourable case, we had only crosslinking reactions, then we could conclude that for all the samples we had crosslinks and not pendant alkyl chains.

\section{Swelling Behaviour of the Gel Fraction}

In order to determine the equilibrium swelling ratio of gel, we have made swelling kinetics in pure water for three different samples (Figure 6) at $20^{\circ} \mathrm{C}$.

At first, we have observed that the swelling ratio was quite stable during $5 \mathrm{~d}$ and then increased hugely. Concurrently, the aspect of gel changed: from brittle, stiff gels, we arrived to very soft, near liquid gels. After $40 \mathrm{~d}$ for the MM30-200 and $65 \mathrm{~d}$ for the HM30-200 and the HM30-100, the swelling ratio reached a maximum. At this time, we visually observed the dissolution of gels. This kind of behaviour was never observed in the literature, unless if the polymer chains or crosslinks are degradable. ${ }^{26}$ In a previous paper we have demonstrated that the ester linkages for hydrophobically modified CMP were stable as a function of time and temperature in water. ${ }^{27}$ So we have concluded that this behaviour was not caused by the breaking of ester links. Then two hypotheses can explain that. First, a possibly bacterial degradation of the polysaccharide chains could be involved in this result, but the addition of an antibacterial agent (sodium azide) has permitted us to invalidate this hypothesis. Then we have thought that our systems had not reached the gelation point i.e. all the polymer chains were not crosslinked, in spite of they really looked like a gel at a macroscopic level.

To check this behaviour, we did the same experiment with the HM30-200 but at different temperatures (Figure 7). We saw that the dissolution of the gels was accelerated with an increase of temperature whereas no change in the swelling ratio was observed at $4{ }^{\circ} \mathrm{C}$ even after $120 \mathrm{~d}$. These experiments showed that the dissolution of the gel fractions was inescapable and accelerated by the temperature.

At first sight, our gels behave like physical gels in spite of the chemical crosslinks; indeed they are dissolved by the action of time and temperature. It is possible that the gelation observed is caused by the combination of physical interactions i.e. hydrogen bonds between carboxylic group or hydrophobic associations and of chemical bindings.

No change in the viscosity of carboxymethylpullulan versus time and temperature is observed, which indicates that no degradation, conformation change of the polysaccharide or associations via hydrogen bonds occurs. ${ }^{27}$

The effect of temperature on hydrophobic associations has been discussed by some authors who worked on hydrophobically modified polymers. But they do not agree: most of them think that the temperature strengthens the hydrophobic interactions, ${ }^{28,29}$ while others claim that it is the opposite. ${ }^{30,31}$ 


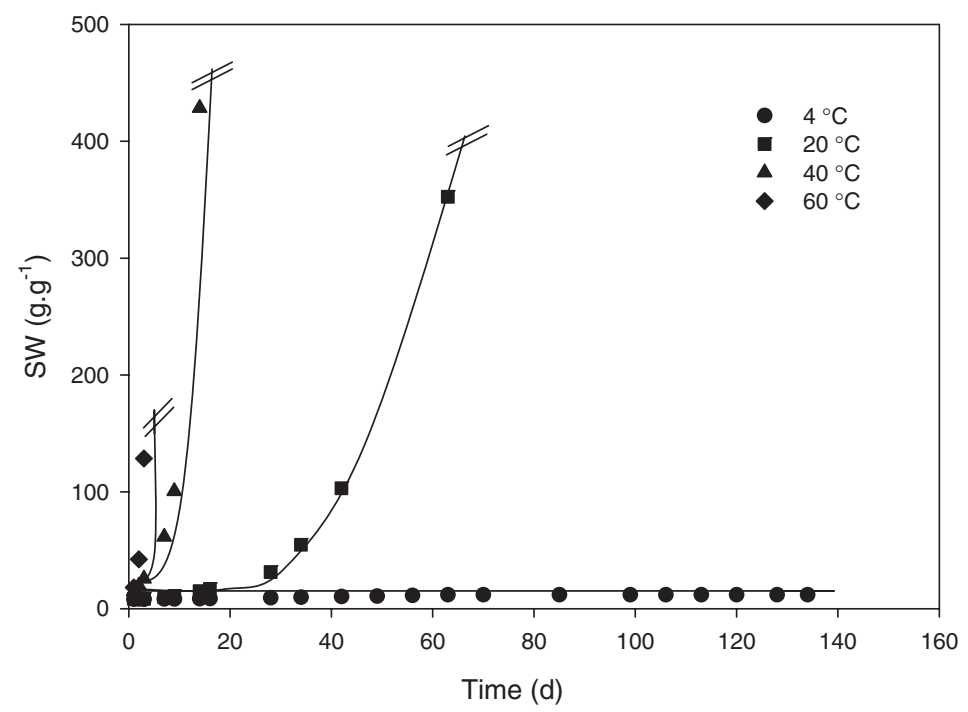

Figure 7. Kinetics of swelling in water of the gel fraction of the HM30-200 as a function of temperature.

Considering our results, our systems act more like the latter, with hydrophobic associations which reorganize as a function of time and temperature.

But we can ask for the utility of the chemical crosslinks in the structure of our products. We have found that the real incorporation ratio of dibromohexan in CMP was about $20 \%$ for all the tested samples, which corresponds to a crosslinking degree of $10 \%$ if all the crosslinks are intermolecular. We could think that the gelation point was reached in comparison with other works where gels were formed with crosslinking degree included between $2 \%$ and $18 \% .{ }^{32-34}$ According to our results, one anhydroglucose unit out of ten was linked; i.e. the number of crosslinks per CMP chains was about 63 for the HMCMP3, 22 for the MMCMP3 and 10 for the LMCMP3. But the theoretical value to reach the gel point was 2 per chains. ${ }^{35}$

In order to explain this behaviour, we have made two hypotheses based on our observations. First, we thought that we had more intramolecular crosslinks than intermolecular ones, what would explain that we have not gelified our systems in spite of the huge quantity of crosslinking agent incorporated. Secondly, because of the kinetics of the crosslinking reaction and the visible heterogeneities, we have considered that we could have obtained highly crosslinked areas leading to dense hydrophobic clusters and low crosslinked areas easy to dismantle.

We have probably the two phenomena combined, but with the above information, it is difficult to settle. In order to understand the structure of our systems, we have to continue this study by physical-chemical analyses on temperature treated hydrogel. Techniques as viscometry, surface tension measurements, quasielastic light scattering and flow field flow fractionation with on line multiangle laser light scattering were used.

\section{CONCLUSION}

Crosslinked carboxymethylpullulans were synthesized with a bifunctional crosslinking agent: the dibromohexan, which presents, thanks to its alkyl chain, a hydrophobic character. We obtained by this reaction on the one hand a heterogeneous gel fraction and on the other hand a sol fraction. It was demonstrated that the sol/gel ratio was dependent on the synthesis conditions $\left(\mathrm{C}_{\mathrm{p}}, \mathrm{R}_{\mathrm{c}}, \bar{M}_{\mathrm{n}}, \ldots\right)$.

Five representative samples were chosen to deepen the structural study. We determined the quantity of dibromohexan incorporated in the polymer and that it reacted by its two brome functions to give only crosslinks.

In studying the behaviour of the gel fraction, we noticed that this fraction was not stable with time and that this phenomenon was stressed with an increase of the temperature.

According to these results, we tried to explain this strange behaviour with two hypotheses: creation of intramolecular crosslinks instead of intermolecular ones or obtaining of highly crosslinked areas held together by hydrophobic associations. We continue with physical-chemical studies to understand the structure and the behaviour of these nanogels.

Acknowledgment. We thank the "French Ministère de l'Education Nationale, de la Recherche et de la Technologie" for financial support. We also thank the "réseau Matériaux, Polymères, Plasturgie (MPP)" and the "région Haute-Normandie" for financial help.

Received: September 21, 2007

Accepted: November 30, 2007

Published: January 17, 2008

\section{REFERENCES}

1. E. Rotureau, M. Leonard, E. Marie, and E. Dellacherie, Colloids Surf., A, 288, 131 (2006).

2. E. Rotureau, E. Marie, M. Leonard, E. Dellacherie, T. A. Camesano, and A. Durand, Colloids Surf., A, 288, 62 (2006).

3. C. Rouzes, M. Leonard, A. Durand, and E. Dellacherie, Colloids 
Surf., B, 32, 125 (2003).

4. C. Lemarchand, R. Gref, C. Passirani, E. Garcien, B. Petri, R. Müller, D. Constantino, and P. Couvreur, Biomaterials, 27, 108 (2006).

5. M. Q. Gu, X. D. Yuan, C. S. Kang, Y. H. Zhao, N. J. Tian, P. Y. Pa, and J. Sheng, Carbohydr. Polym., 67, 417 (2007).

6. N. Venkatesan and S. P. Vyas, Int. J. Pharm., 203, 169 (2000).

7. M. Leonard, M. Rastello de Boisseson, P. Hubert, F. Dalençon, and E. Dellacherie, J. Controlled Release, 98, 395 (2004).

8. R. Rodriguez, C. Alvarez-Lorenzo, and A. Concheiro, Eur. J. Pharm. Sci., 20, 429 (2003).

9. L. Noble, A. I. Gray, L. Sadiq, and I. F. Uchegbu, Int. J. Pharm., 192, 173 (1999).

10. K. Akiyoshi, S. Deguchi, N. Moriguchi, S. Yamaguchi, and J. Sunamoto, Macromolecules, 26, 3062 (1993).

11. T. Nishikawa, K. Akiyoshi, and J. Sunamoto, J. Am. Chem. Soc., 118, 6110 (1996).

12. K. Akiyoshi, S. Kobayashi, S. Shichibe, D. Mix, M. Bardy, S. W. Kim, and J. Sunamoto, J. Controlled Release, 54, 313 (1998).

13. K. Ikeda, T. Okada, S. I. Sawada, K. Akiyoshi, and K. Matsuzaki, FEBS Lett., 580, 6587 (2006).

14. K. Na, T. B. Lee, K. H. Park, E. K. Shin, Y. B. Lee, and H. K. Choi, Eur. J. Pharm. Sci., 18, 165 (2003).

15. S. Vinogradov, E. Batrakova, and A. Kabanov, Colloids Surf., B, 16, 291 (1999).

16. S. Vinogradov, E. V. Batrakova, and A. Kabanov, Bioconjugate Chem., 15, 50 (2004).

17. S. Vinogradov, E. Kohli, and A. D. Zeman, Mol. Pharmaceutics, 2, 449 (2005).

18. V. Dulong, D. Le Cerf, L. Picton, and G. Muller, Colloids Surf., A, 274, 163 (2006).

19. T. Coviello, M. Grassi, G. Rambone, E. Santucci, M. Carafa, E.
Murtas, F. M. Riccieri, and F. Alhaique, J. Controlled Release, 60, 367 (1999).

20. T. Coviello, M. Grassi, G. Rambone, and F. Alhaique, Biomaterials, 22, 1899 (2001).

21. S. Simon, J. Y. Dugast, D. Le Cerf, L. Picton, and G. Muller, Polymer, 44, 7917 (2003).

22. C. Duval, D. Le Cerf, L. Picton, and G. Muller, J. Chromatogr. B 753, 115 (2000).

23. R. W. Eyler, T. S. Klug, and F. Siephuis, Anal. Chem., 19, 24 (1947).

24. S. V. Vinogradrov, T. K. Bronich, and A. V. Kabanov, Adv. Drug Delivery Rev., 54, 135 (2002).

25. V. Crescenzi, A. Francescangeli, A. Taglienti, D. Capitani, and L. Mannina, Biomacromolecules, 4, 1045 (2003).

26. A. S. Hoffman, Adv. Drug Delivery Rev., 43, 3 (2002).

27. S. Simon, G. Mocanu, L. Picton, D. Le Cerf, and G. Muller, e-polymers, 38, 1 (2004).

28. C. Creuzet, S. Kadi, M. Rinaudo, and R. Auzily-Velty, Polymer, 47, 2706 (2006).

29. J. Desbrières, C. Martinez, and M. Rinaudo, Int. J. Biol. Macromol., 19, 21 (1996).

30. E. Volpert, J. Selb, and F. Candau, Polymer, 39, 1025 (1998).

31. S. Biggs, J. Selb, and F. Candau, Polymer, 34, 580 (1993).

32. V. Crescenzi, A. Francescangeli, D. Capitani, L. Mannina, D. Renier, and D. Bellini, Carbohydr. Polym., 53, 311 (2003).

33. D. Capitani, A. A. De Angelis, V. Crescenzi, G. Masci, and A. L. Segre, Carbohydr. Polym., 45, 245 (2001).

34. S. C. Chen, Y. C. Wu, F. L. Mi, Y. H. Lin, L. C. Yu, and H. W. Sung, J. Controlled Release, 96, 285 (2004).

35. P. Matricardi, M. Dentini, V. Crescenzi, and S. B. Ross-Murphy, Carbohydr. Polym., 27, 215 (1995). 